\title{
EL SIGNIFICADO DE LA FÓRMULA "SER DEL COMIENZO" EN LA CIENCIA DE LA LÓGICA DE HEGEL
}

\section{Jorge Eduardo Fernández*}

La fórmula "ser del comienzo"1 es utilizada por Hegel para denominar al ser inmediato indeterminado a partir del cual debe ser hecho el comienzo de la ciencia. En ella convergen comienzo (Anfang-Arché) y ser. Esto significa que para Hegel el ser es la única y fáctica respuesta a la pregunta por el comienzo. El ser del comienzo no es ni se representa mediante ningún principio, él es pura determinidad (Bestimmtheit).

Hegel inicia el primer pasaje de la Ciencia de la lógica con la pregunta: "Con qué tiene que ser becho el comienzo de la ciencia?"2. Este pasaje ocupa un curioso lugar en la obra pues no pertenece a la Introducción y tampoco está contenido en la organización de sus secciones y capítulos. Él aclara que esta pregunta surge en torno al debate ${ }^{3}$, contemporáneo suyo, acerca de si es posible hallar un comienzo de la filosofía, y si éste debe ser mediato o inmediato. La respuesta más contundente que formula Hegel a esta dificultad es la siguiente: "Nada bay en el cielo, en la naturaleza, en el espiritu o dónde sea, que no contenga al mismo tiempo la inmediatezy la mediación"A .

Es decir que el comienzo deberá ser tan inmediato como mediato. Es más, comenzar significa mediar la inmediatez. En el primer capítulo de la Doctrina del ser Hegel califica al ser como inmediato e indeterminado.

* Profesor en la Univ. Nac. de San Martín (UNSAM). jorgeedufer@fibertel.com.ar ${ }^{1}$ La fórmula "Sein des Anfangs" es utilizada por Hegel por única vez al inicio del capítulo dedicado al ser-para-sí.

${ }^{2}$ W. L., p. 53/87. 'Womit muss der Anfang der Wissenschaft gemacht werden?"

${ }^{3} \mathrm{La}$ referencia es al debate entre Jacobi y Schelling generado en torno a la obra de Jacobi: Von den Göttlichen Dingen. Tenemos que considerar aquí que el planteo acerca de la inmediatez del comienzo tuvo una significativa repercusión en el pensamiento de Hegel. Al respecto se puede leer: Gawoll, H. J.: 'Der logische Ort des Wabren. Jacobi und Hegels Wissenschaft vom Sein", pp. 90 ss..

${ }^{4}$ W.L., 54/88. "...nichts im Himmel oder in der Natur oder im Geiste oder wo es sei, was nicht ebenso die Unmittelbarkeit enbält als die Vermittlung....”. 
Comienzo y ser están relacionados de tal manera que en su determinidad se encuentra la raíz del desarrollo especulativo del despliegue de "lo lógico".

Hegel muestra cómo la pregunta por el comienzo precede y abarca a la pregunta por el ser ${ }^{5}$, y que el comenzar, en tanto principio (Prinzip) y resolución (Entschlu $\beta$ ), opera el vaciamiento de contenido del saber y pone al ser puro en su inmediatez. El "ser del comienzo" es el ser puro vaciado de contenido y significado puesto como signo del comienzo, nombra pues el lado puesto o positivo del comenzar.

Todo esto nos conduce a tener que considerar en el ser los distintos aspectos que constituyen el comienzo y que traspasan con él. Esto implica en principio, reconocer la permanencia del comienzo en el ser, la cual queda expresada en la fórmula "ser del comienzo". El comienzo es conservado en el ser como clave del comenzar, pero es conservado en tanto ser puesto mediante el comenzar. En la misma acción de comenzar el comienzo es puesto en cuanto ser: El ser y el hacer del comienzo son uno. Esto significa que el ser es la única y fáctica respuesta a la pregunta por el comienzo y que sólo mediante el ser el comienzo traspasa. "El ser puro bace el comienzo",

El comienzo sólo puede ser puesto en la determinidad de ser, con lo cual la "determinidad" no remite entonces al contenido determinado puesto como fundamento o principio, sino al ser inmediato e indeterminado. El ser del comienzo no es ni se representa mediante ningún principio, él es determinidad pura.

Consecuentemente Hegel inicia la Doctrina del ser con la sección que lleva el título: "Determinidad". "Determinidad" es el título que abarca toda la Primera sección, es decir, es aplicado tanto al ser, como al existir y al serpara-sí. Estrictamente hablando la Ciencia de la lógica no comienza con la

${ }^{5} \mathrm{Al}$ respecto puede leerse el escrito de Milan Prucha: Seinsfrage und Anfang in Hegels Wissensfft der Logik., pp. 109 ss.. En este escrito se radicaliza la posición ante este tema sosteniendo: "Die Frage nach dem Anfang der Logik ist für Hegel die Frage nach dem Sein", p. 109. Aunque es difícil sostener esta proposición tal cual está formulada, pienso que sí se puede sostener como queda dicho en nuestro texto, que la pregunta por el ser sin ser la pregunta por el comienzo queda comprendida en ella. La diferencia puede resultar capital cuando debamos pensar la unidad de comienzo y ser, y también al tener que sostener la diferencia entre ambos.

${ }^{6}$ Enc.: $§$ 86. 'Das reine sein macht den Anfang....". 
unidad de ser y nada, sino con el parágrafo dedicado a la determinidad. La determinidad es la primera significación de la cualidad y señala la esfera en la cual ser, existir y ser-para-sí son pensados.

Por primera vez en la historia de la filosofía el comienzo de la ciencia es hecho a partir de la cualidad. El ser del comienzo es el ser cualitativo. La determinaciones cualitativas preceden a la cuantitativas, y estas últimas se deben derivar de las primeras y no a la inversa. Dice Hegel:

"Quizá sólo pueda observarse todavía, que la determinación de cantidad se antepone por lo demás a la de cualidad, y eso -como en la mayoría de los casos- sin mayor fundamento. Ya se mostró como el comienzo se efectúa con el ser como tal, y por consiguiente con el ser cualitativo. De la comparación de la cantidad con la cualidad resulta fácilmente evidenciado que por su naturaleza aquella es primera. Pues la cantidad es la cualidad ya convertida en negativa; la magnitud es la determinación que ya no es uno con el ser, sino diferente de él, es la cualidad determinada que se convirtió en indiferente". [... ...] "La cualidad por lo tanto, como determinación inmediata, es primera, y con ella debe hacerse el comienzo"7.

El tratamiento del comienzo le permitió a Hegel concretar uno de los cambios más significativos de la filosofía de su tiempo: comenzar la Ciencia de la lógica a partir de la inmediatez cualitativa del ser. Esto significa en primer término devolver a la filosofía a su ámbito propio. Esta devolución implica un retorno, pero un retorno a través del cual la filosofía también se transforma. Dicha transformación recae no sólo en la estructura de la Ciencia de la lógica, sino también en su método. La filosofía moderna, dependiente en su método de otras ciencias, retorna a su suelo original: el ser. Ahora bien, este retornar es un anticipar, un paso hacia atrás, hacia el comienzo, en lo que respecta a las determinaciones cuantitativas. Es en sentido cabal un

${ }^{7}$ W.L., p. 66-67/102. 'Nur dies kann etwa bemmerkt werden, daß sonst die Bestimmung de Quantität vor der Qualität aufgefübrt wird, -und dies-wie das meiste- obne weiteren Grund. E ist bereits gezeigt worden, daß der Anfang sich mit dem Sein als solchem macht, daber mit dex. quelitativen Sein. Aus der Vergleichung der Qualität mit der Quatität erbellt leicht, daß jene di der Natur nach erste ist. Denn die Quantität ist die schon negativ-gewordene Qualität; die Gri ist die Bestimmtheit, die nicht mebr mit dem Sein eins, sondern schon von ibm unterschieden, d aufgehobene, gleichgültig gewordene Qualität ist". [...] "Die Qualität ist daber als a unmittelbare Bestimmtheit die erste, und mit ibr der Anfang zu machen". 
horadar los principios en busca del comenzar. Esta anticipación de la determinaciones cuantitativas implica poner en marcha una profunda transformación de la metafísica moderna. Esta transformación es, en sentido especulativo, una anticipación, un retorno hacia el comienzo como nofundamento del fundamento como ámbito que precede a todas las determinaciones posteriores.

Este ámbito o esfera que precede a la cantidad es la cualidad. El tratamiento del ser cualitativo, desarrollado no sólo en el pasaje sobre el comienzo, sino también en toda la Primera sección de la Doctrina del ser, anticipa los presupuestos de la cantidad y de la magnitud que como señala Hegel son posteriores y se derivan de ella.

Toda la Primera sección de la Doctrina del ser se encuentra presentada por un parágrafo cuyo título es, como antes indicamos, "Determinidad", y puesto entre paréntesis: "Cualidad"8.

Ahora bien, este desarrollo se encuentra contenido en la esfera de la determinidad. Debemos empezar aclarando que traducir la palabra alemana "Bestimmheit" por determinidad conlleva una seria dificultad. Por un lado es un término clave mediante el cual se expresa la significatividad del "ser del comienzo", y por otro lado la palabra "determinidad" no aparece en los diccionarios de lengua castellana ${ }^{\text {. }}$

Hecha esta ineludible aclaración, recordemos que la determinidad nos introduce en la consideración del ser en general, del puro ser o ser del comienzo, del cual dice Hegel:

"El ser es lo inmediato indeterminado; está libre de la determinidad respecto de la esencia, así como está libre de aquella que puede alcanzar en el interior de él mismo. Este ser carente de reflexión es el ser tal como es de modo inmediato sólo en él.

${ }^{8} \mathrm{La}$ relación entre determinidad y cualidad que queda encerrada en tal paréntesis es algo que sólo puede comprenderse desde la relación entre Sein y Dasein, pues el significado de cualidad que empieza a tratarse aquí, se desarrolla a lo largo de los tres capítulos que componen la Primera sección de la Doctrina del ser.

9 En coincidencia con Ramón Valls Plana opté por traducir Bestimmtheit por "determinidad". Según se sigue de textos del mismo Hegel la palabra determinidad traduce la griega "horos" y la latina "determinatio", lo que permite concebir a la determinidad como la expresión inmediata del límite cualitativo. 
El significado de la fórmula "Ser del comienzo" en la ciencia de la lógica de Hegel -103

Porque es indeterminado, es un ser desprovisto de cualidad; pero el carácter de la indeterminidad le compete en sí sólo en oposición a lo determinado o cualitativo. Pero al ser en general se le contrapone el ser determinado como tal; pero de este modo su indeterminación misma constituye su cualidad" 10 .

La Ciencia de la lógica comienza entonces con la determinidad, Hegel se refiere en este primer parágrafo al ser en cuanto tal o determinidad. En ella, la contradicción inmediata del ser es puesta en la forma especulativa de la negación de la negación. El ser, el absoluto, previo a toda determinación, es determinado como inmediato e indeterminado.

La contraposición queda puesta de manifiesto pues el primer parágrafo que lleva el título "Determinidad" habla del ser libre de toda determinidad. Este estar libre de toda determinidad, aclara Hegel, lo es tanto respecto de las determinaciones de la esencia como de las de su propio interior. Es el ser carente de toda determinación o cualidad al cual sólo se le pueden adjudicar la indeterminación y la inmediatez en oposición a la determinación y a la mediación. Sólo podemos pensar el ser desde la esfera finita del existir o ser determinado. El ser del comienzo es "lo inmediato indeterminado", es decir es el ser puro, libre de toda determinación. Es el ser carente de reflexión, tanto externa como interna. Pero la indeterminidad, en tanto "carácter" que le "compete en sí", sólo puede ser afirmada desde la oposición con el ser determinado o ser cualitativo. Esto significa en principio, que el ser es carente de cualidad, pero esta carencia de cualidad sólo puede ser pensada como indeterminidad dentro de la esfera de la determinidad. Con ello la determinidad queda concebida como el límite negativo-positivo mediante el cual el ser del comienzo significa. Determinidad es entonces el límite negativo-positivo de la cualidad en donde la indeterminidad como carácter del ser es determinada en cuanto tal.

Hacer el comienzo implica de este modo mostrar la contradicción interna al ser. Del puro ser del comienzo sólo se puede hablar de manera negativa y desde la negación ya implícita en su oposición respecto al ser

10 W.L., p. 68-69/105. 'Das Sein ist das unbestimmte Unmittelbare; es ist frei von der Bestimmtheit gegen das Wesen sowie noch von jeder, die es innerhalb seiner selbst erbalten kann. Diese Reflexionslose Sein ist das Sein, wie es unmittelbar nur an ibm selber ist.

Weil es unbestimmt ist, ist es qualitätsloses Sein; aber an sich kommt ibm der Charakter der Unbestimmtheit nur im Gegensatz gegen das Bestimmte oder Qualitative $z^{\prime \prime}$. 
determinado. Esta instancia negativa del lenguaje respecto del ser del comienzo, que expresa su carencia de cualidad, sólo adquiere significado en oposición a lo determinado o cualitativo, es decir como negación de la oposición vuelta posición en el pensamiento especulativo. Pensar especulativamente es determinar lo indeterminado, poner el límite como negación de la negación, desarrollar el ser en la esfera de la determinidad (el horos) ${ }^{11}$.

A continuación del último párrafo que hemos citado, Hegel indica el camino a recorrer en esta primera sección. Primero "Hay que mostrar que el primer ser está determinado", que su indeterminidad es su determinación. En segundo lugar mostrar que el ser, mediante este contener la determinación en sí mismo, traspasa al existir. Y en tercer lugar que el ser finito se niega a sí mismo en cuanto tal y traspasa al ser-para-sí que es el ser en tanto referencia infinita hacia sí mismo. Con este programa traza Hegel el desarrollo de la esfera de la determinidad, la esfera del desarrollo de la referencialidad inherente al ser.

Dicho en otros términos, mediante la Doctrina del ser se efectúa el traspaso de lo infinito a lo finito, necesidad que surge de las limitaciones de la metafísica formal, las cuales Hegel ha dejado planteadas en el desarrollo de las Posiciones del pensamiento ante la objetividad ${ }^{12}$. Según hemos anticipado en dicho punto, en tanto Spinoza era juzgado de panteísta por diluir la substancia en los atributos, la metafísica formal, presa de una comprensión cuantitativa de la determinación, no puede llegar a pensar la unidad de la substancia y los atributos. $\mathrm{Al}$ anticipar las determinaciones cuantitativas mediante la cualidad, Hegel introduce la diferencia cualitativa en el orden de las determinaciones; mediante la cualidad desobtura esta confrontación planteada en el núcleo de la filosofía del siglo XVIII ${ }^{13}$.

11 Ya en el Prólogo a la Fenomenología del espíritu Hegel habla de la determinidad utilizando el témino griego "boros" para referirse a la doble negación implícita en el límite del concepto. PhG.: p. 14. Vers. Cast.: p. 11

${ }^{12}$ Enzylklopädie der Philosopbischen Wissenschaften. (1830). § 36 al $\$ 83$.

13 Enc.: Nota al $\ 36$. Hablando de la contradicción entre el ser y sus atributos, interna a la metafísica moderna, Hegel concluye: "Al llegar a este punto, esa contradicción sólo permite la vaporosa solución de empujarla basta lo carente de determinación, basta el sensum eminentiorem, mediante un aumento cuantitativo. Pero con la solución el atributo es efectivamente 
Hegel llama cualidad a la esfera de la determinidad en la cual es concebido el traspasar de lo infinito a lo finito, la cual será considerada finalmente como la determinidad infinita de lo finito o ser-para-sí. El paso principal que da Hegel mediante este traspaso, es el de introducir la diferencia en las determinaciones cuantitativas de la metafísica formal, y con ella el momento positivo de la especulación.

Hegel empieza el primer capítulo de la Doctrina del ser con el mismo estilo despojado del parágrafo precedente. Sin artículos que connoten esencias, sin verbos que introduzcan acción.

"Ser, puro ser, -sin ninguna otra determinación".

El ser inmediato indeterminado es el ser puro, donde "puro" significa vacío de determinación. El ser indeterminado y puro, es el puro intuir, pensar vacío, nada. Como habíamos señalado, el ser, supuesto de toda ciencia, vaciado de todo contenido mediante el trabajo del comienzo, es el ser puesto en su inmediatez e indeterminación.

En el $\int 19$ de la Enciclopedia Hegel define a la Lógica como: "...la ciencia pura de la idea"; allí puro remite a "...la idea en el elemento abstracto del pensar". Abstracto y puro adquieren significaciones muy semejantes; abstracto califica al ser liberado de toda otra determinación que no sean aquellas que provienen de la naturaleza de su elemento, es decir, del pensar.

De este modo se puede comprender por qué Hegel inicia la Doctrina del ser en la Enciclopedia diciendo:

“El ser es el concepto sólo en sí; ...”"14.

¿Qué relación hay entre los parágrafos de la Lógica grande y estos d $\epsilon$ la Enciclopedia? Si con la definición del $\ 19$ podemos comenzar a saber qué es la Lógica, con la del $\ 84$ empezamos a definir qué es "lo lógico". "Lc lógico" es la idea puesta en el elemento puro del pensar. Ser es entonces la significación inmediata de la idea puesta en dicho elemento, "...el concepto sól

aniquilado y reducido a mera nombre". 'Dieser Widerspruch läßt auf diesem Standpunkte nur dì nebulose Auflösung durch quantitative Steigerung zu, sie ins Bestimmungslose, in den sensum eminentioren zu treiben. Hierdurch aber wird die Eigenschaft in der Tat zunichte gemacht und ibs blo $\beta$ ein Name gelassen.

14 Enc.: \84. 'Das Sein ist der Begriff nur an sich...". 
en si;..." que opera como supuesto y clave de toda otra ulterior determinación.

Ahora bien, en tanto en la gran Lógica el ser es considerado desde su oposición con el ser determinado o existir, en la Enciclopedia es definido desde su relación con la idea. El ser en tanto es la idea puesta en su inmediatez e indeterminación en el elemento abstracto del pensar, es "el concepto sólo en si".

En esta definición se encuentra contenida la simple y compleja contradicción del ser, del pensar y por cierto también del lenguaje. La misma se expresa comúnmente diciendo que ser es el más pobre y general de los conceptos, y por eso no podemos decir de él nada más que es, o como sucede con Hegel, sólo podemos hablar del ser del comienzo desde su opuesto, el existir.

Todo lo que se puede decir es ser, así empieza la interpretación de este pasaje Anton $\mathrm{Koch}^{15}$; también hace lo mismo Pirmin SteckelerWeithofer desde su teoría crítica de la significación. Allí se señala:

"Concepto "en si" es la aparente inmediata referencia de palabras, proposiciones o algunas representaciones semióticas, las cuales llegan a ser puestas en principio como puras referencias formales, es decir, como momentos abstractos de significación. $\mathrm{El}$ análisis del ser, de la lógica del ser, se ocupa esencialmente de la crítica de cada hipóstasis madura de la significación formal, la cual es constitutiva interna en cuanto tal de un sistema usual de un lenguaje general, o demanda de signos" ${ }^{\prime 16}$.

Lo primero a saber es si este sutil y complicado poner en significación admite una mejor manera que no sea esta mediación especulativa del comienzo. En lo que se refiere al trabajo de Pirmin Stekeler-Weithofer cabe cuestionarse si el despliegue de la cosa misma se expresa a través de una postura analítica y crítica. $O$ lo que es lo mismo, hay que preguntarse si desde este tipo de análisis se llega a mostrar la puesta en movimiento del pensamiento especulativo a partir de la contradicción inherente al concepto

${ }^{15}$ Koch, A.: Sein - Nicht-Werden. En: Hegels Seinslogik. Arndt, A./Iber, Ch. (Hg.). Akademie. Berlin. 2000, p. 141.

${ }^{16}$ Pirmin Stekeler-Weithofer: Hegels Analytische Philosophie. Die Wissenschaft der Logik als kritische Theorie der Bedeutung. F. Schöningh. Paderborn. 1992. p. 95. 
en sí, o sólo se alcanza legítimamente a representar un proceso de sucesivas determinaciones.

Ahora bien, lo que me interesa destacar del párrafo recién citado, es la significatividad del ser puro. Ser en tanto concepto en sí es signo puro, carente de significación, y en esta carencia radica la "demanda de signos". El signo puro opera como clave del comienzo en tanto "demanda de signos".

La fundamentación de esta concepción del ser en tanto signo inmediato del concepto la podemos encontrar en los parágrafos de la Enciclopedia dedicados a la representación" ${ }^{17}$. La "demanda de signos" surge de esta tensión interna a la inteligencia expresada por Hegel mediante la imagen del "pozo oscuro en el que se guarda un mundo infinito"18. De este modo la representación es la unidad de la inteligencia en tanto ésta representa "algo otro". El signo "es la pirámide en la cual se ba colocado un alma extraña y la cobija"19 Esta unidad de lo extraño con lo propio se da mediante el signo. El ser es signo del comienzo en tanto une lo lógico y la lógica. En esta tensión inherente al signo se expresa pues lo que Hegel llama, de manera cabal y depurada, el "instinto lógico'no del lenguaje. Por ello ser sólo puede ser vaciado desde dentro, la caída de ningún otro signo lo denota.

En esta dirección se encuentra también el comentario de Biard, J.; Buvat, J-F. y otros:

"Ser es el concepto más pobre de la Ciencia de la lógica. Estrictamente hablando él no merece ser llamado concepto: del ser en cuanto tal, no se puede decir nada, pues el es pura vacuidad"21.

${ }^{17}$ Fue Jacques Derrida quien propuso una lectura semiológica de la Lógica de Hegel. Ver: El pozo y la pirámide, en: Márgenes de la filosofía, Cátedra. Madrid, 1989, pp. 103 a 144.

18 Enc.: Notal al \$453. 'Die Intelligenz als diesen Nácbtlichen Schacht, in welchem ein Welt unendlich vieler Bilder und Vorstellungen aufbewabrt ist...".

19 Enc.: Notal al \$458. “...die Pyramide, in welche eine frende Seele versetz und aufbewabrt ist".

${ }^{20}$ Enc.: Notal al $\$ 459$. “... logische Instinkt...”.

${ }^{21}$ Biard, J.; Buvat, J-F. y otros: "Introduction à la lecture de La Science de la Logique de Hegel", p. 47. "'Etre' est le concept le plus pauvre de la Science de la Logique. A strictement parler, il ne mérite mème pas le titre de concept: de l'etre en tant que tel, on ne peut rien dire, car il est pure vacuite". 
¿Si ser no merece ser llamado concepto, entonces cómo llamarlo?

La esfera de la cualidad contiene al ser en su inmediatez indeterminación, es decir en su determinidad. La determinidad del $\mathrm{s}$ significa su inmediata e indeterminada posición en el elemento abstracto d pensar. Pura significatividad en la cual su carencia de cualidad es su cualida su indeterminidad es su determinidad. La demanda de significado está prefigurada en la pura significatividad del ser, que es signo o concepto sólo en sí. Ser es significación del concepto vacío ${ }^{22}$, signo de la experiencia de la no experiencia.

Siguiendo este planteo llegamos a comprender que el trabajo del comienzo ha vaciado lo lógico como concepto para ponerlo como signo. Ser es el concepto puesto como signo.

En este sentido podemos decir que la Ciencia de la lógica contiene la metafísica y su crítica, en tanto la analogía y el análisis están contenidos como momentos negativos de la dialéctica. Pero la dialéctica contiene a su vez el momento positivo característico de lo especulativo. Según palabras del mismo Hegel:

"Lo especulativo o racional-positivo aprehende la unidad de las determinaciones en su oposición, lo afirmativo que se contiene en la disolución de ellas y en su pasar" ${ }^{23}$.

Lo especulativo señala el traspaso como el movimiento racionalpositivo. El traspasar de las determinaciones del ser, en tanto concepto en sí, tiene "su determinación más precisa" en la forma de lo dialéctico ${ }^{24}$. Lo dialéctico en tanto despliegue especulativo del ser es el desarrollo más preciso de las determinaciones simples del concepto.

${ }^{22} \mathrm{La}$ existencia de conceptos vacíos de experiencia es la cuestión con la que Kant inicia los Principios de una deducción trascendental en general. Ver en especial: $\mathrm{KrV}$.: B 117. "Tch nenne daber die Erklärung der Art, wie sich Begriffe a priori auf Gegenstände beziehen können, die transzendentale Deduktion..."

${ }^{23}$ Enc.: $\int 82$. "Das Spekulative oder Positiv-Vernünftige faßt die Einheti der Bestimmungen in ibrer Entgegensetzung auf, das Affirmative, das in ibrer Auflösung und ibrem übergehen entbalten ist". ${ }^{24} \mathrm{Ib}$., $§ 84$. 
Como ya hemos citado, en el Prólogo a la Fenomenología, en los parágrafos dedicados a la proposición especulativa, Hegel se refiere a estas determinaciones llamándolas "automovimientos puros" o "almas"25.

La Doctrina del ser realiza esta tarea, la de desarrollar las "almas" del concepto, las cuales determinan la flexión interna al ser. Al cual, en tanto puesto en el elemento abstracto del pensar le corresponde el desarrollo de las determinaciones simples del concepto, "el ser en sí, para sí, la igualdad consigo mismo, etc,...”. El ser, único signo subsistente en sí, pone en el pensar la contradicción interna al concepto. Su generalidad y su pobreza inician el movimiento especulativo como proceso de significación $\mathrm{y}$ conceptualización.

La afirmación de Aristóteles: "El ser se dice de muchas maneras" sigue valiendo aquí siempre y cuando coloquemos el acento en la distinción y contradicción entre la unidad implícita del "se dice" y la multiplicidad de significados. "El ser se dice de muchas maneras" porque "el ser se dice". El ser significa, es decir, es puro signo y demanda de signos. Este decirse del ser coloca a la determinidad o cualidad como determinación inmediata al ser. Por ello esta pura significatividad del ser y su inmediata demanda del concepto, son pensados en la esfera de la cualidad, o si se lo quiere decir con la precisión de la gran Lógica, en la esfera de la determinidad. La determinidad está designando la esfera lógica en donde el ser es puesto en su inmediatez e indeterminación. En ella se efectúa el comienzo positivo del desarrollo en cuanto el ser, como signo del comienzo, es puesto como concepto en sí.

Así cobra sentido la primera afirmación de la Enciclopedia comprendida bajo el título cualidad:

"El ser puro hace el comienzo, porque tanto como pensamiento puro como también indeterminado, es simple inmediatez, pues el primer comienzo no puede ser nada mediato y menos aún determinado"26.

25 PhG., p. 42. Ver nota: 80.

${ }^{26}$ Enc. \86. 'Das reien Sein macbt den Anfang, weil es sowobl reiner Gedanke als das unbestimmte, einfache unmittelbare ist, der erste Anfang aber nicbts Vermitteltes und weiter Bestimmtes sein kann". 
El comienzo se hace mediante el ser puro porque su inmediatez reúne la indeterminación de su carácter objetivo con el concepto en sí. Tal inmediatez expresa la ausencia de reflexión en el comienzo. Por ello Hegel critica los puntos de partida de las filosofías de Fichte y de Schelling, indicando que el $\mathrm{Yo}=\mathrm{YO}$ y la indiferencia absoluta o la identidad respectivamente, implican una reflexión y por lo tanto no son lo primero. Sólo el ser en su inmediatez e indeterminación es "pensar puro e intuir".

La tarea de depuración y acción del comienzo constituye para Hegel el momento de consumación de la filosofí. El "ser del comienzo" es el "puro ser" (reine Sein) o ser vacío (leere Sein) puesto como signo puro carente de significación. El ser no es el comienzo, más el comienzo debe ser hecho con el ser, pues en su pureza y vacuidad de significación el ser es demanda de signos. "Ser del comienzo" expresa al ser en tanto signo puro.

Carencia y significación, considerados hasta aquí como negación y posición del ser del comienzo, se consuman ambos simultánea y conjuntamente. La consumación del escepticismo conlleva también la del nominalismo. Todas las palabras o representaciones pueden ser vaciadas de significado y puestas en función de la pura significación. Pero el ser puro puede ser considerado, en el contexto de la Doctrina del ser, como el resultado irreductible de la prueba de resistencia semiótica efectuada por el comienzo. El escepticismo se consuma en el comienzo. "Ser" es el único signo que significa aún vacío de significados, es el único signo significante en sí.

\section{Resumen}

La fórmula "ser del comienzo" es utilizada por Hegel para denominar al ser inmediato indeterminado a partir del cual debe ser hecho el comienzo de la ciencia. En ella convergen comienzo (Anfang-Arché) y ser. El ser y el hacer del comienzo son uno. Esto significa para Hegel que el ser es la única y fáctica respuesta a la pregunta por el comienzo. El ser del comienzo no es ni se representa mediante ningún principio, él es pura determinidad (Bestimmtheit).

La tarea de depuración y acción del comienzo constituye para Hegel el momento de consumación de la filosofía. "Ser" en tanto concepto en sí es puesto como signo puro vaciado de significación. Pero - en esta carencia de significación radica la "demanda de signos". "Ser del comienzo" expresa al ser en tanto signo puro.

Carencia y significación, considerados hasta aquí como negación y posición del ser del comienzo, se consuman ambos simultánea y conjuntamente. La consumación del escepticismo conlleva también del nominalismo. Todas las palabras o 
representaciones pueden ser vaciadas de significado y puestas en función de la pura significación. Pero el ser puro puede ser considerado, en el contexto de la Doctrina del ser, como el resultado irreductible de la prueba de resistencia semiótica efectuada por el comienzo. El escepticismo se consuma en el comienzo. "Ser" es el único signo que significa aún vacío de significados, es el único signo significante en sí. Palabras clave: "Lógica", "ser", “comienzo".

\section{Abstract}

The formula "being of beginning " is employed by Hegel to name the immediate nondetermined being from which the beginning of science must take place. Beginning (Anfang, Arkbe) come together in it. Being and doing of beginning are one. To Hegel, this means that being is the only and factic response to the question about beginning. The being of beginning neither is nor represents itself by means of any principle, it is pure "determinetness".

To Hegel, the task of depuration and action of beginning constitutes the moment of consummation of philosophy. "Being", as a concept in itself, is set as pure sign void of signification. But in this lack of signification lies the "demand of signs". "Being of beginning" expresses being as pure sign.

Lack and significance, considered up to now as negation and position of the being of beginning, are both consummated simultaneously and at the same time. Consummation of scepticism also carries nominalism with it. All words and representations may be emptied of significance and set to work in view of pure signification. But pure being may be considered, in the context of the Doctrine of Being, as the irreductible result of the proof of semiotic resistance carried out in the beginning. Scepticism consummates itself in beginning. "Being" is the only sign that signifies, even empty of significations; it is the only signifier in itself.

Key words: "Logics”, 'Being”, "beginning”. 